\title{
Effects of Radiation and Porosity of the Medium on MHD Flow Past an Inclined Plate in the Presence of Hall Current
}

\author{
U.S. Rajput, G. Kumar \\ Department of Mathematics and Astronomy, University of Lucknow, India \\ E-mail: rajputgauravlko@gmail.com
}

Received: 09 January 2017; revised: 10 April 2017; accepted: 24 April 2017; published online: 30 June 2017

\begin{abstract}
The present study deals with an analysis of the effects of radiation and porosity of the medium on unsteady natural convection flow of a viscous, incompressible and electrically conducting fluid past an impulsively started inclined plate under the influence of transversely applied uniform magnetic field and Hall current. The medium of the flow is taken as porous. The governing equations involved in the present analysis are solved by the Laplace-transform technique. The results obtained are discussed with the help of graphs drawn for different parameters like thermal Grashof number, mass Grashof Number, Prandtl number, Hall current, radiation, permeability, magnetic field parameter and Schmidt number. The numerical values obtained for skin-friction and Nusselt number have been tabulated. The objective of this study is to analyze radiation and the porosity of the medium in the MHD flow model. In the study we found that the velocity in the boundary layer increases with the values of radiation and porosity of the medium. It is also observed that radiation and porosity of the medium increases the skin-friction at the wall surface. The results of the study are found to be in agreement with the actual flow. The importance of the problem can be seen in cooling of electronic components of a nuclear reactor, bed thermal storage and heat sink in the turbine blades.
\end{abstract}

Key words: MHD flow, porous medium, radiation effect, mass diffusion, Hall current

\section{INTRODUCTION}

The MHD flow with heat and mass transfer plays an important role in different areas of science and technology like chemical engineering, mechanical engineering, biological science, petroleum engineering, biomechanics, irrigation engineering and aerospace technology. Study of radiation with heat transfer and mass diffusion is essential in describing several fluid models. The effect of radiation in free convection from a vertical porous plate was studied by Hossain et al. [5]. Wilhem and Choi [16] have considered magnetohydrodynamic diffusion flow across homogeneous magnetic field. Raptis [7] has considered radiation and free convection flow through a porous medium. Further, Raptis along with Perdikis [8] have worked on unsteady flow through a highly porous medium in the presence of radiation. The radiation effect on combined convection over a vertical flat plate embedded in a porous medium of variable porosity was presented by Pal and Mondal [6]. Vyas and Srivastava [14] have analyzed radiative MHD flow over a non isothermal stretching sheet in a porous medium. Rajput and Sahu [9] have investigated the radiation effect on steady hydromagnetic flow of a viscous fluid through a vertical channel in a porous medium with heat generation or absorption. The radiation effect on MHD flow past an infinite vertical plate with variable temperature and uniform mass diffusion was analyzed by Deka and Deka [3]. Radiation and mass transfer effects on transient free convection flow of a dissipative fluid past semi-infinite vertical plate with uniform heat and mass flux was investigated by Vasu et al. [13]. Influence of thermal radiation on a transient MHD Couette flow through a porous medium was considered by Baoku et al. [2]. Armstrong and Muthucumaraswamy [1] have worked on MHD flow past a parabolic started vertical plate with variable temperature and mass diffusion. In MHD flows, if applied magnetic field is of high strength on the flow then Hall current on MHD flow is also significant. Some papers related with combined effects of Hall current and radiation are also mentioned here. Guchhait et al. [4] have investigated combined effects of Hall current and radiation on MHD free convective flow in a vertical chan- 
nel with an oscillatory wall temperature. Thamizhsudar and Pandurangan [12] have worked on combined effects of radiation and Hall current on MHD flow past an exponentially accelerated vertical plate in the presence of rotation. Effects of induced magnetic field and homogeneous-heterogeneous reactions on stagnation flow of a Casson fluid was investigated by Raju et al [11]. Combined effects of Hall current and magnetic field on unsteady flow past a semi infinite vertical plate with thermal radiation and heat source was studied by Srihari [11]. The chemical reaction effect on unsteady MHD flow past an impulsively started oscillating inclined plate with variable temperature and mass diffusion in the presence of Hall current was analyzed by us [10]. In this paper an analysis of radiation effect on unsteady MHD flow through porous medium past an impulsively started inclined plate with variable temperature and mass diffusion in the presence of Hall current has been done. The problem is solved analytically using the Laplace Transform technique. A selected set of graphical results illustrating the effects of various parameters involved in the problem are presented and discussed. The numerical values of skin-friction and Nusselt number have been tabulated.

\section{MATHEMATICAL ANALYSIS}

The geometric model of the problem is shown in Fig. 1.

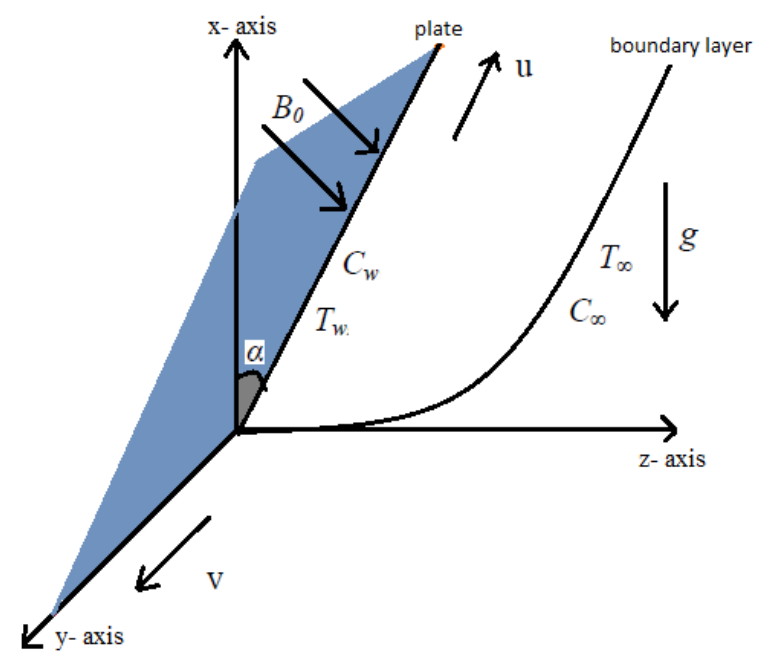

Fig. 1. Physical model

An electrically non conducting plate inclined at an angle $\alpha$ from vertical is considered here. $x$ axis is taken along the vertical plane and $z$ normal to it. A transverse magnetic field $B_{0}$ of uniform strength is applied on the flow. Initially it has been considered that the plate as well as the fluid is at the same temperature $T_{\infty}$. The species concentration in the fluid is taken as $C_{\infty}$. At time $t>0$, the plate starts moving with a velocity $u_{0}$ in its own plane, and temperature of the plate is raised to $T_{w}$. The concentration $C$ near the plate is raised linearly with respect to time.

The flow model is as under:

$$
\begin{gathered}
\frac{\partial u}{\partial t}=v \frac{\partial^{2} u}{\partial z^{2}}+g \beta \operatorname{Cos} \alpha\left(T-T_{\infty}\right) \\
+g \beta^{*} \operatorname{Cos} \alpha\left(C-C_{\infty}\right)-\frac{\sigma B_{0}^{2}(u+m v)}{\rho\left(1+m^{2}\right)}-\frac{v \mathrm{u}}{\mathrm{K}} \\
\frac{\partial v}{\partial t}=v \frac{\partial^{2} v}{\partial z^{2}}+\frac{\sigma B_{0}^{2}(m u-v)}{\rho\left(1+m^{2}\right)}-\frac{v v}{K} \\
\frac{\partial C}{\partial t}=D \frac{\partial^{2} C}{\partial z^{2}} \\
\rho C_{p} \frac{\partial T}{\partial t}=k \frac{\partial^{2} T}{\partial z^{2}}-\frac{\partial q_{r}}{\partial z}
\end{gathered}
$$

The boundary conditions taken are as under:

$$
\left\{\begin{array}{l}
t \leq 0: u=0, v=0, T=T_{\infty} C=C_{\infty} \text { for every } z \\
t>0: u=u_{0}, v=0, T=T_{\infty}+\left(T_{w}-T_{\infty}\right) A \\
C=C_{\infty}+\left(C_{w}-C_{\infty}\right) A, \text { at } z=0 \\
u \rightarrow 0, \quad v \rightarrow 0, \quad T \rightarrow T_{\infty} C \rightarrow C_{\infty} \text { as } z \rightarrow \infty
\end{array}\right.
$$

The local radiant in equation (4), for the case of an optically thin gray gas, is expressed by

$$
\frac{\partial q_{r}}{\partial z}=-4 a^{*} \sigma\left(T_{\infty}^{4}-T^{4}\right)
$$

Considering the temperature difference within the flow sufficiently small, $T^{4}$ can be expressed as the linear function of temperature. This is accomplished by expanding $T^{4}$ in a Taylor series about $T_{\infty}$ and neglecting higher-order terms

$$
T^{4} \cong 4 T_{\infty}^{3} T-3 T_{\infty}^{4}
$$

Using equations (6) and (7), equation (4) becomes

$$
\rho C_{p} \frac{\partial T}{\partial t}=k \frac{\partial^{2} T}{\partial z^{2}}-16 a^{*} \sigma T_{\infty}^{3}\left(T-T_{\infty}\right)
$$

The following non-dimensional quantities are introduced to transform equations (1), (2), (3) and (8) into dimensionless 
form:

$$
\begin{gathered}
\overline{\mathrm{z}}=\frac{\mathrm{zu}_{0}}{v}, \overline{\mathrm{u}}=\frac{\mathrm{u}}{\mathrm{u}_{0}}, \overline{\mathrm{v}}=\frac{\mathrm{v}}{\mathrm{u}_{0}}, G_{r}=\frac{g \beta v\left(T_{w}-T_{\infty}\right)}{u_{0}^{3}}, \\
\mathrm{P}_{\mathrm{r}}=\frac{\mu \mathrm{c}_{\mathrm{p}}}{\mathrm{k}}, \mathrm{R}=\frac{16 \mathrm{a}^{*} \sigma v^{2} T_{\infty}^{3}}{k u_{0}^{2}}, \theta=\frac{\left(\mathrm{T}-\mathrm{T}_{\infty}\right)}{\left(\mathrm{T}_{\mathrm{w}}-\mathrm{T}_{\infty}\right)}, \\
\mathrm{M}=\frac{\sigma \mathrm{B}_{0}^{2} v}{\rho \mathrm{u}_{0}^{2}}, \mathrm{~S}_{\mathrm{c}}=\frac{v}{\mathrm{D}}, \mathrm{G}_{\mathrm{m}}=\frac{\mathrm{g} \beta^{*} v\left(\mathrm{C}_{\mathrm{w}}-\mathrm{C}_{\infty}\right)}{\mathrm{u}_{0}^{3}} \\
\mu=\rho v, \bar{K}=\frac{u_{0}^{2}}{v^{2}} K, \overline{\mathrm{C}}=\frac{\left(\mathrm{C}-\mathrm{C}_{\infty}\right)}{\left(\mathrm{C}_{\mathrm{w}}-\mathrm{C}_{\infty}\right)}, \bar{t}=\frac{\mathrm{tu}_{0}^{2}}{v} .
\end{gathered}
$$

Then the model becomes

$$
\begin{gathered}
\frac{\partial \bar{u}}{\partial \bar{t}}=\frac{\partial^{2} \bar{u}}{\partial \bar{z}^{2}}+G_{r} \cos \alpha \theta \\
+G_{m} \operatorname{Cos} \alpha \bar{C}-\frac{M(\bar{u}+m \bar{v})}{1+m^{2}}-\frac{1}{\bar{K}} \bar{u} \\
\frac{\partial \bar{v}}{\partial \bar{t}}=\frac{\partial^{2} \bar{u}}{\partial \bar{z}^{2}}+\frac{M(m \bar{u}-\bar{v})}{1+m^{2}}-\frac{1}{\bar{K}} \bar{v} \\
\frac{\partial \bar{C}}{\partial \bar{t}}=\frac{1}{S_{c}} \frac{\partial^{2} \bar{C}}{\partial \bar{z}^{2}} \\
\frac{\partial \theta}{\partial \bar{t}}=\frac{1}{P_{r}} \frac{\partial^{2} \theta}{\partial \bar{z}^{2}}-\frac{R \theta}{P_{r}}
\end{gathered}
$$

The corresponding boundary conditions become

$$
\left\{\begin{array}{l}
\bar{t} \leq 0: \overline{\mathrm{u}}=0, \overline{\mathrm{v}}=0, \theta=0, \overline{\mathrm{C}}=0, \text { for every } \bar{z} \\
\bar{t}>0: \bar{u}=1, \bar{v}=0, \theta=\bar{t} \bar{C}=\bar{t}, \text { at } \bar{z}=0 \\
\overline{\mathrm{u}} \rightarrow 0, \overline{\mathrm{v}} \rightarrow 0, \quad \theta \rightarrow 0, \overline{\mathrm{C}} \rightarrow 0, \quad \text { as } \bar{z} \rightarrow \infty
\end{array}\right.
$$

Dropping bars in the above equations, we get

$$
\begin{gathered}
\frac{\partial u}{\partial t}=\frac{\partial^{2} u}{\partial z^{2}}+G_{r} \cos \alpha \theta \\
+G_{m} \cos \alpha C-\frac{M(u+m v)}{1+m^{2}}-\frac{1}{\mathrm{~K}} \mathrm{u} \\
\frac{\partial v}{\partial t}=\frac{\partial^{2} v}{\partial z^{2}}+\frac{M(m u-v)}{1+m^{2}}-\frac{1}{K} v \\
\frac{\partial C}{\partial t}=\frac{1}{S_{c}} \frac{\partial^{2} C}{\partial z^{2}} \\
\frac{\partial \theta}{\partial t}=\frac{1}{P_{r}} \frac{\partial^{2} \theta}{\partial z^{2}}-\frac{R \theta}{P_{r}}
\end{gathered}
$$

The boundary conditions are

$$
\left\{\begin{array}{l}
t \leq 0: u=0, v=0, \theta=0, C=0, \text { for every } z \\
t>0: u=1, v=0, \theta=t, C=t, \text { at } z=0 \\
u \rightarrow 0, v \rightarrow 0, \quad \theta \rightarrow 0, C \rightarrow 0, \quad \text { as } z \rightarrow \infty
\end{array}\right.
$$

Combining equations (15) and (16), the model becomes

$$
\frac{\partial q}{\partial t}=\frac{\partial^{2} q}{\partial z^{2}}+G_{r} \cos \alpha \theta+G_{m} \cos \alpha C-q a
$$

$$
\begin{gathered}
\frac{\partial C}{\partial t}=\frac{1}{S_{c}} \frac{\partial^{2} C}{\partial z^{2}}, \\
\frac{\partial \theta}{\partial t}=\frac{1}{P_{r}} \frac{\partial^{2} \theta}{\partial z^{2}}-\frac{R \theta}{P_{r}} .
\end{gathered}
$$

The boundary conditions now become:

$$
\left\{\begin{array}{l}
t \leq 0: q=0, \theta=0, C=0, \text { for every } z \\
t>0: q=1, \theta=t, C=t, \quad \text { at } z=0 \\
q \rightarrow 0, \quad \theta \rightarrow 0, C \rightarrow 0, \quad \text { as } z \rightarrow \infty
\end{array}\right.
$$

Here $q=u+i v, a=\frac{M(1-i m)}{1+m^{2}}+\frac{1}{K}$.

The dimensionless governing equations (20) to (22), subject to the boundary conditions (23), are solved by the usual Laplace - transform technique. The solution of the model is as under:

$$
\begin{aligned}
q & =\frac{1}{2} e^{-\sqrt{a} z} A_{33}+\frac{G_{r} \cos \alpha}{4(a-R)^{2}}\left[2 e^{-\sqrt{a} z}\left(A_{1}+P_{r} A_{2}\right)\right. \\
& +2 t A_{2} e^{-\sqrt{a} z}(a-R)+z A_{3} e^{-\sqrt{a} z}\left(\sqrt{a}-\frac{R}{\sqrt{a}}\right) \\
& \left.+2 A_{12} A_{4}\left(1-P_{r}\right)\right]+\frac{G_{m} \cos \alpha}{4 a^{2}}\left[e^{-\sqrt{a} z}\left(2 A_{1}+2 \sqrt{a} A_{3}\right)\right. \\
& \left.+2 e^{-\sqrt{a} z} A_{2}\left(S_{c}+a t\right)+2 A_{13} A_{5}\left(1-S_{c}\right)\right] \\
& -\frac{P_{r} G_{r} \cos \alpha}{2 \sqrt{\pi}(a-R)^{2} A_{11}}\left[A_{16} A_{6} \sqrt{\pi} z\left(a t-1-R t+P_{r}\right)\right. \\
& \left.+A_{14} A_{7} \sqrt{\pi} z\left(1-P_{r}\right)+\frac{1}{2} \sqrt{\frac{P_{r}}{R}} A_{16} A_{8} A_{11} \sqrt{\pi} z(a-R)\right] \\
& -\frac{G_{m} \cos \alpha}{2 a^{2} \sqrt{\pi}}\left[2 a z \sqrt{t S_{c}} e^{-\frac{z^{2} S_{c}}{4 t}}\right. \\
& \left.+A_{15} \sqrt{\pi}\left(a z^{2} S_{c}+2 a t+2 S_{c}-2\right)+A_{13} \sqrt{\pi}\left(A_{9}+A_{10} S_{c}\right)\right] . \\
& C=t\left\{\left(1+\frac{z^{2} S_{c}}{2 t}\right) \operatorname{erfc}\left[\frac{\sqrt{S_{c}}}{2 \sqrt{t}}\right]-\frac{z \sqrt{S_{c}}}{\sqrt{\pi \sqrt{t}}} e^{-\frac{z^{2}}{4 t}} S_{c}\right\},
\end{aligned}
$$


Tab. 1. Skin friction for different parameters ( $\alpha$ in degree)

\begin{tabular}{c|c|c|c|c|c|c|c|c|c|c|c}
\hline$\alpha$ & $M$ & $m$ & $P r$ & $S c$ & $G m$ & $G r$ & $R$ & $K$ & $t$ & $\tau_{x}$ & $\tau_{y}$ \\
\hline 15 & 2 & 1 & 0.71 & 2.01 & 100 & 10 & 2 & 0.2 & 0.2 & 3131.81 & 0457.75 \\
\hline 30 & 2 & 1 & 0.71 & 2.01 & 100 & 10 & 2 & 0.2 & 0.2 & 2807.64 & 0410.42 \\
\hline 60 & 2 & 1 & 0.71 & 2.01 & 100 & 10 & 2 & 0.2 & 0.2 & 1619.92 & 0237.03 \\
\hline 30 & 3 & 1 & 0.71 & 2.01 & 100 & 10 & 2 & 0.2 & 0.2 & 2565.71 & 0535.13 \\
\hline 30 & 5 & 1 & 0.71 & 2.01 & 100 & 10 & 2 & 0.2 & 0.2 & 2144.10 & 0673.74 \\
\hline 30 & 2 & 3 & 0.71 & 2.01 & 100 & 10 & 2 & 0.2 & 0.2 & 3205.23 & 0306.42 \\
\hline 30 & 2 & 5 & 0.71 & 2.01 & 100 & 10 & 2 & 0.2 & 0.2 & 3283.74 & 0203.80 \\
\hline 30 & 2 & 1 & 7.00 & 2.01 & 100 & 10 & 2 & 0.2 & 0.2 & -0301.65 & 03.6341 \\
\hline 30 & 2 & 1 & 0.71 & 3.00 & 100 & 10 & 2 & 0.2 & 0.2 & 2807.40 & 0410.42 \\
\hline 30 & 2 & 1 & 0.71 & 4.00 & 100 & 10 & 2 & 0.2 & 0.2 & 2807.20 & 0410.41 \\
\hline 30 & 2 & 1 & 0.71 & 2.01 & 010 & 10 & 2 & 0.2 & 0.2 & 2805.66 & 0410.40 \\
\hline 30 & 2 & 1 & 0.71 & 2.01 & 050 & 10 & 2 & 0.2 & 0.2 & 2806.54 & 0410.41 \\
\hline 30 & 2 & 1 & 0.71 & 2.01 & 100 & 50 & 2 & 0.2 & 0.2 & 14039.5 & 2051.31 \\
\hline 30 & 2 & 1 & 0.71 & 2.01 & 100 & 100 & 2 & 0.2 & 0.2 & 28079.4 & 4102.41 \\
\hline 30 & 2 & 1 & 0.71 & 2.01 & 100 & 10 & 3 & 0.2 & 0.2 & 3261.74 & 0533.73 \\
\hline 30 & 2 & 1 & 0.71 & 2.01 & 100 & 10 & 5 & 0.2 & 0.2 & 4651.50 & 0963.89 \\
\hline 30 & 2 & 1 & 0.71 & 2.01 & 100 & 10 & 2 & 0.5 & 0.2 & 4652.18 & 0963.92 \\
\hline 30 & 2 & 1 & 0.71 & 2.01 & 100 & 10 & 2 & 1.0 & 0.2 & 5740.74 & 1341.06 \\
\hline 30 & 2 & 1 & 0.71 & 2.01 & 100 & 10 & 2 & 0.2 & 0.3 & 4812.72 & 0856.91 \\
\hline 30 & 2 & 1 & 0.71 & 2.01 & 100 & 10 & 2 & 0.2 & 0.4 & 6849.75 & 01348.0 \\
\hline
\end{tabular}

$$
\begin{aligned}
& \theta=\frac{e^{-\sqrt{R} z}}{4 \sqrt{R}}\left\{\operatorname{erfc}\left[\frac{-2 \sqrt{R} t+z P_{r}}{\sqrt{P_{r} t}}\right](2 \sqrt{R} t-z R)+e^{2 \sqrt{R} z}\right. \\
& \left.\operatorname{erfc}\left[\frac{2 \sqrt{R} t+z P_{r}}{\sqrt{P_{r} t}}\right](2 \sqrt{R} t+z R)\right\} .
\end{aligned}
$$

The expressions for the symbols involved in the above solution are given in the appendix.

\section{SKIN FRICTION}

The dimensionless skin friction at the plate $z=0$ is obtained by

$$
\left(\frac{d q}{d z}\right)_{z=0}=\tau_{x}+i \tau_{y}
$$

The numerical values of $\tau_{x}$ and $t a u_{y}$, for different parameters are given in Tab. 1.

\section{NUSSELT NUMBER}

The dimensionless Nusselt number at the plate $z=0$ is given by

$$
\begin{aligned}
N u & =\left(\frac{\partial \theta}{\partial z}\right)_{z=0}=\operatorname{erfc}\left[\frac{\sqrt{R} t}{\sqrt{t P_{r}}}\right]\left(\sqrt{R} t-\frac{\sqrt{R}}{2} t+\frac{P_{r}}{4 \sqrt{R}}\right) \\
& -\operatorname{erfc}\left[-\frac{\sqrt{R} t}{\sqrt{t P_{r}}}\right]\left(\frac{\sqrt{R}}{2} t+\frac{P_{r}}{4 \sqrt{R}}\right)-\frac{e^{-\frac{R t}{P_{r}}} \sqrt{t P_{r}}}{\sqrt{\pi}} .
\end{aligned}
$$

The numerical values of $\mathrm{Nu}$, for different parameters are given in Tab. 2.

Tab. 2. Nusselt number for different parameters

\begin{tabular}{c|c|c|c}
\hline$P r$ & $R$ & $t$ & $N u$ \\
\hline 0.71 & 2 & 0.4 & -0.805273 \\
\hline 7.00 & 2 & 0.4 & -1.959260 \\
\hline 0.71 & 3 & 0.4 & -0.894014 \\
\hline 0.71 & 4 & 0.4 & -0.976083 \\
\hline 0.71 & 2 & 0.5 & -0.950956 \\
\hline 0.71 & 2 & 0.6 & -1.094940 \\
\hline
\end{tabular}




\section{RESULT AND DISCUSSIONS}

The velocity profiles for different parameters are shown in Figures 2 to 21. Temperature profiles for different values of $\operatorname{Pr}, R$ and time are shown in Figures 22 to 24. It is observed from Figures 2 and 12 that the primary and secondary velocities of fluid decrease when the plate angle $(\alpha)$ is increased. This is in agreement with the actual flow, since the velocity of the fluid decreases as we increase the inclination of the plate from the vertical. Figures 3, 13, 4 and 14 show the buoyancy effect. It is observed that both the primary and secondary velocities increase on increasing mass Grashof number $(\mathrm{Gm})$ and thermal Grashof number $(G r)$. This indicates that the buoyancy force in the boundary layer region near the plate tends to accelerate primary and secondary velocities. Further, Figures 5 and 15 show the influence of Hall current on primary and secondary velocities, respectively. Primary velocity increases rapidly near the surface of the plate, whereas secondary velocity decreases throughout the boundary layer region on the increasing Hall current parameter $(m)$. This shows that Hall current tends to accelerate primary velocity in the region near the surface of the plate, whereas it tends to retard secondary velocity in the boundary layer. It is observed from Figures 6 and 16 that the effect of increasing values of the parameter $M$ results in decreasing $u$ and increasing $v$. It is in agreement, since the magnetic field establishes a force which acts against the main flow resulting in slowing down the velocity of the fluid. It is deduced that velocities are increased when radiation parameter $R$ is increased (Figures $7,17)$. It indicates that the effect of radiation parameter on the fluid in the boundary layer region near the plate tends to accelerate the velocities. From Figures 8 and 18, it is observed that increasing the permeability parameter $(K)$ of the porous medium, the primary velocity and the secondary velocity increase. This is because an increase in $(K)$ implies that there is a decrease in the resistance of the porous medium which tends to accelerate primary velocity as well as secondary velocity in the boundary layer region. Further, it is observed that velocities decrease when Prandtl number and Schmidt number are increased (figures 9, 19, 10 and 20). In actual sense, the increase of $S c$ means a decrease of molecular diffusivity $(D)$. This shows that the process of diffusion will decrease. Further, from Figures 11 and 21, it is observed that velocities increase with time. It is observed that temperature decreases when Prandtl the number and radiation parameter are increased (Figures 22 and 23). However, from Figure 23 it is observed that temperature increases with time.

Skin friction is given in Tab. 1. The value of $\tau_{x}$ increases with the increase in thermal Grashof number, mass Grashof Number, Hall current parameter, radiation parameter, permeability parameter and time; and it decreases with the angle of inclination of the plate, the magnetic field, Prandtl number and Schmidt number. A similar effect is observed with $\tau_{y}$, except magnetic field and Hall parameter, in which case $\tau_{y}$ increases with magnetic field parameter, and decreases with Hall parameter.

The Nusselt number is given in Tab. 2. The value of $N_{u}$ decreases with increase in Prandtl number, radiation parameter and time.

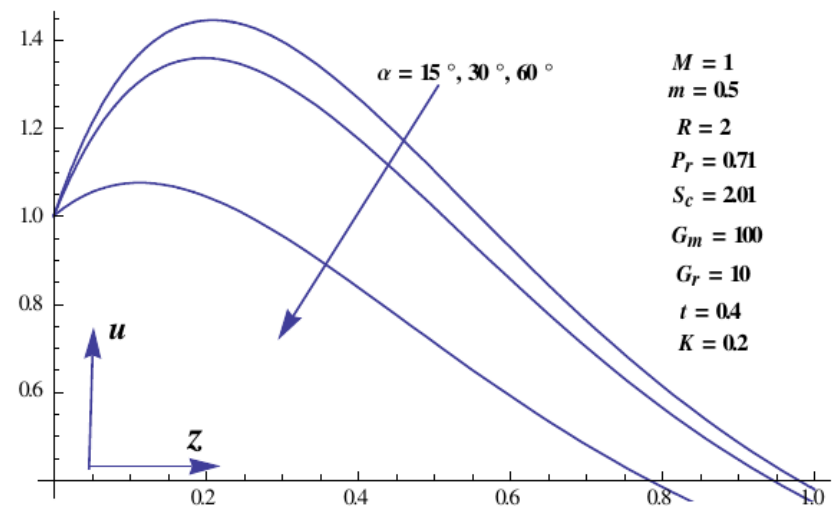

Fig. 2. Velocity $u$ for different values of $\alpha$

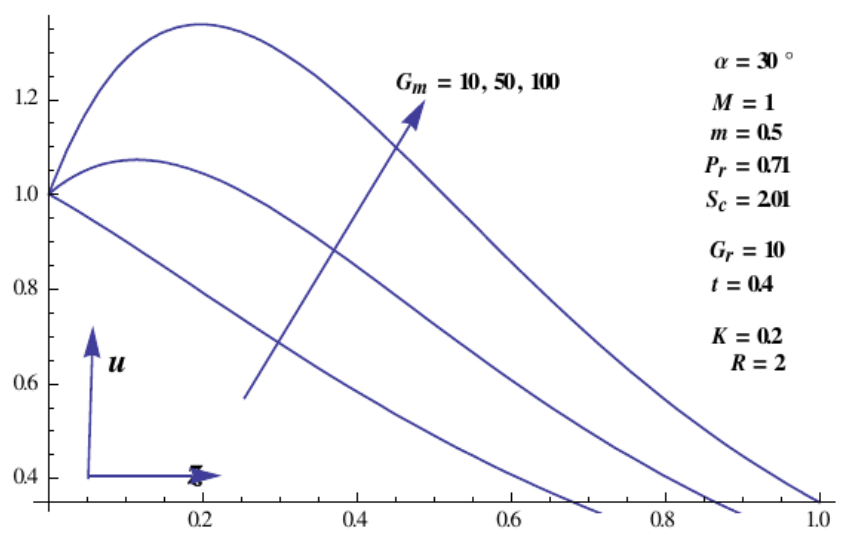

Fig. 3. Velocity $u$ for different values of $G m$

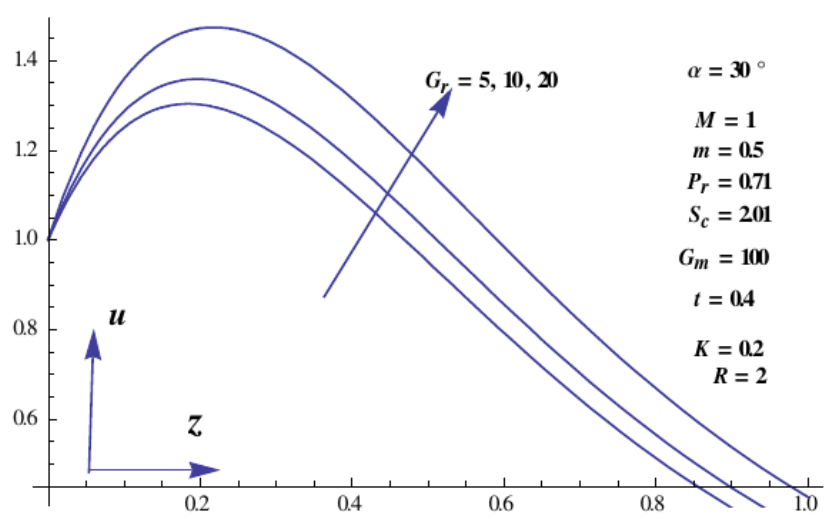

Fig. 4. Velocity $u$ for different values of $G r$ 


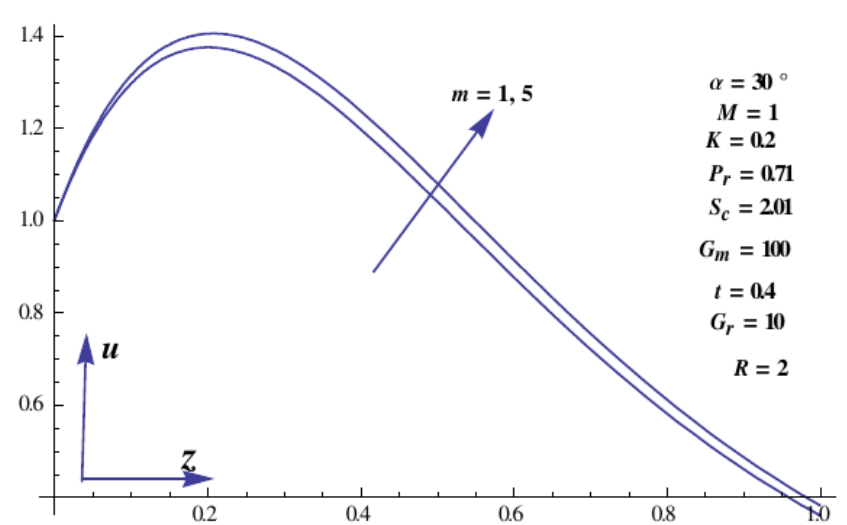

Fig. 5. Velocity $u$ for different values of $m$

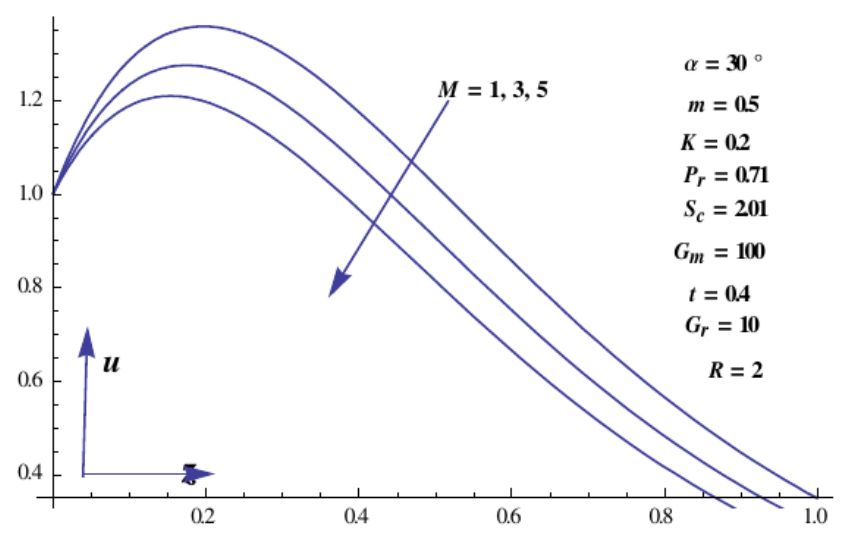

Fig. 6. Velocity $u$ for different values of $M$

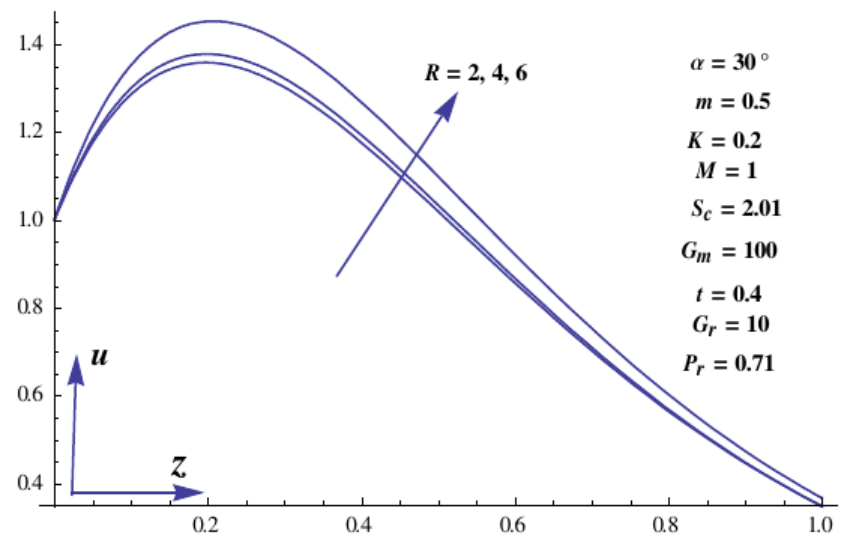

Fig. 7. Velocity $u$ for different values of $R$

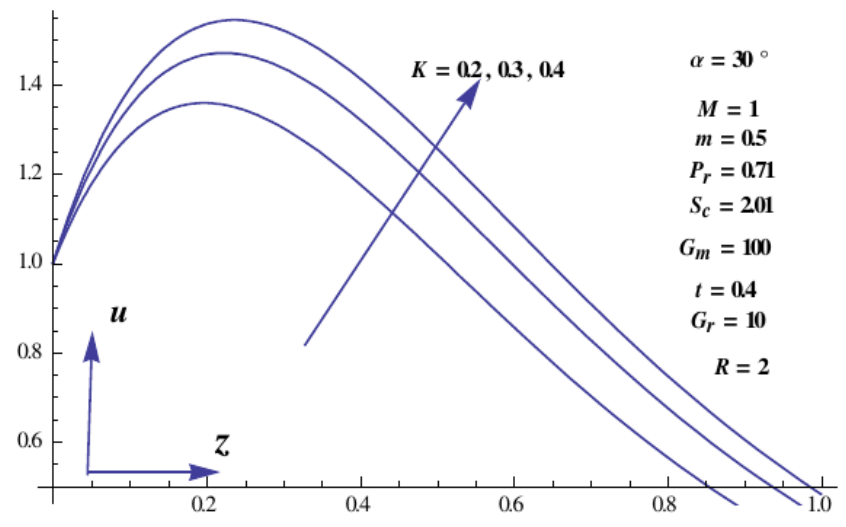

Fig. 8. Velocity $u$ for different values of $K$

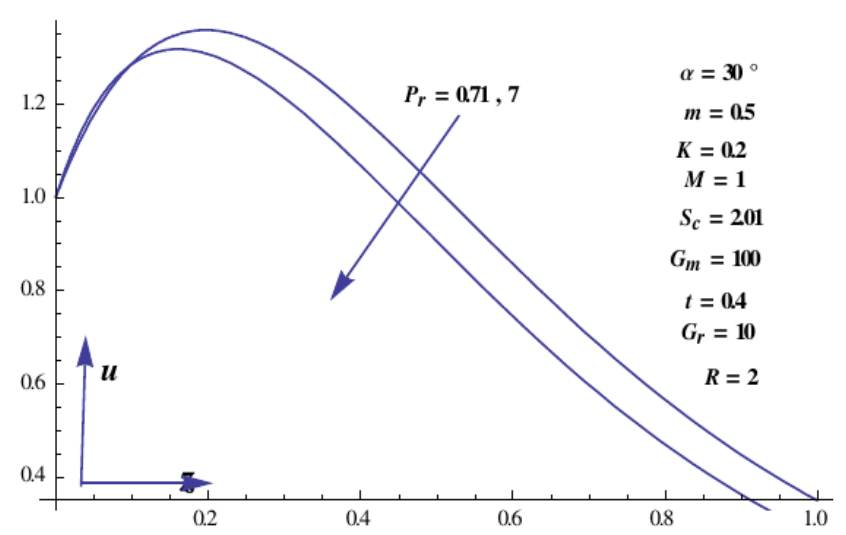

Fig. 9. Velocity $u$ for different values of $\mathrm{Pr}$

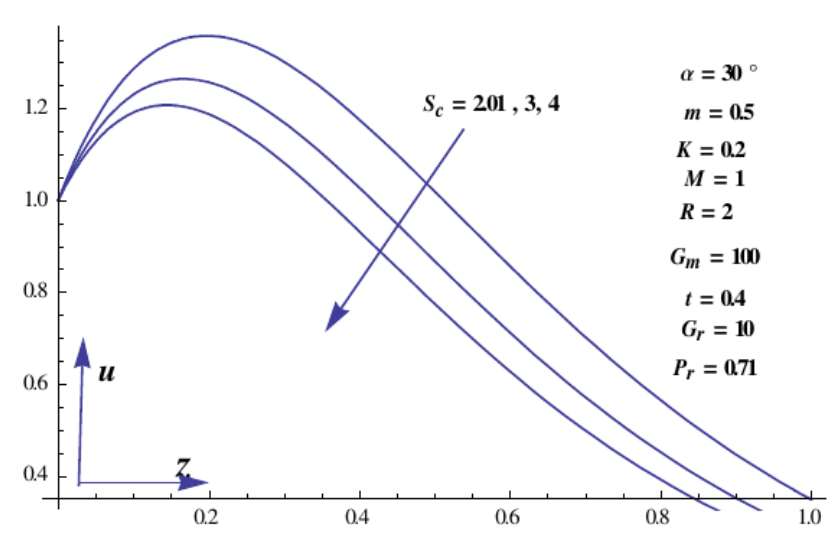

Fig. 10. Velocity $u$ for different values of $S c$ 


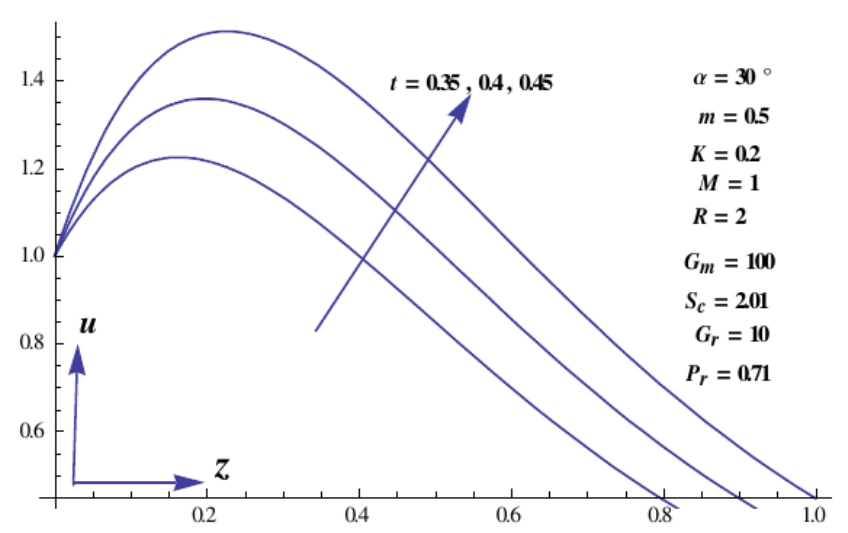

Fig. 11. Velocity $u$ for different values of $t$

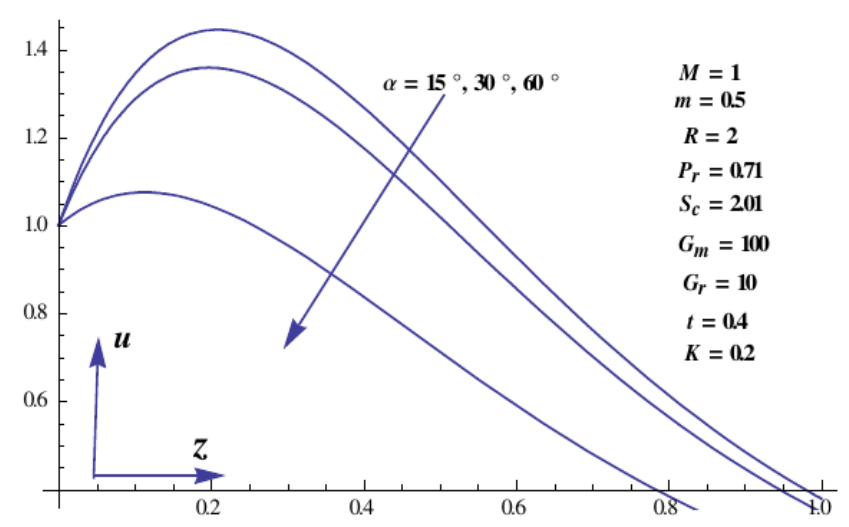

Fig. 12. Velocity $v$ for different values of $\alpha$

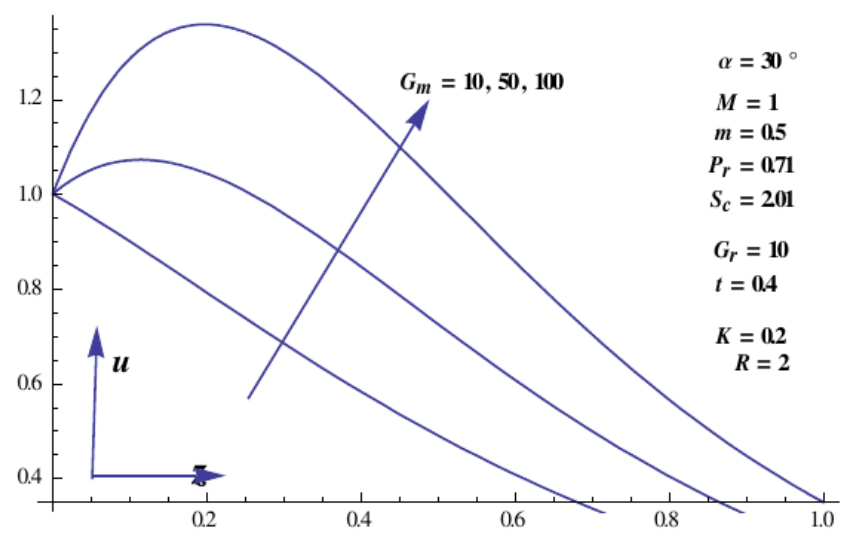

Fig. 13. Velocity $v$ for different values of $G m$

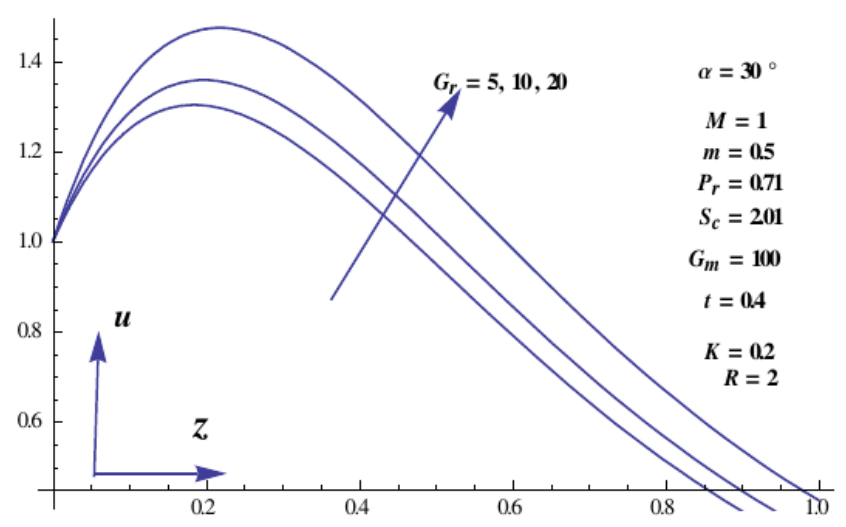

Fig. 14. Velocity $v$ for different values of $G r$

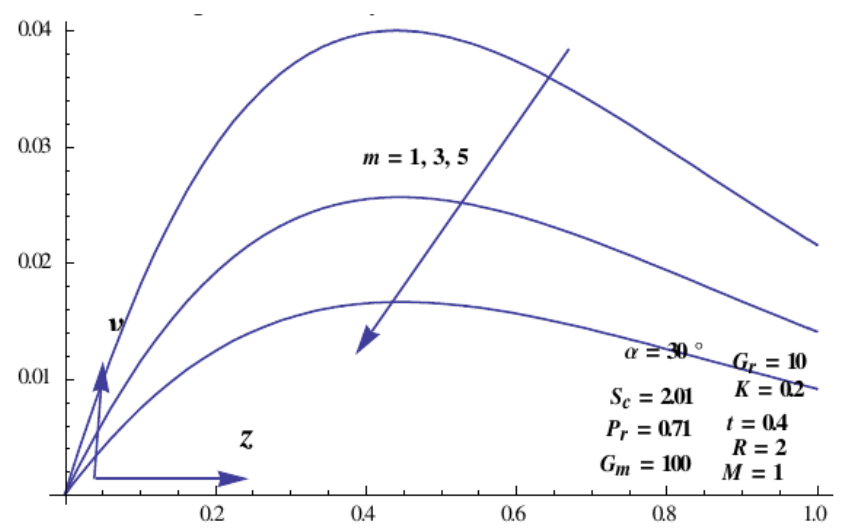

Fig. 15. Velocity $v$ for different values of $m$

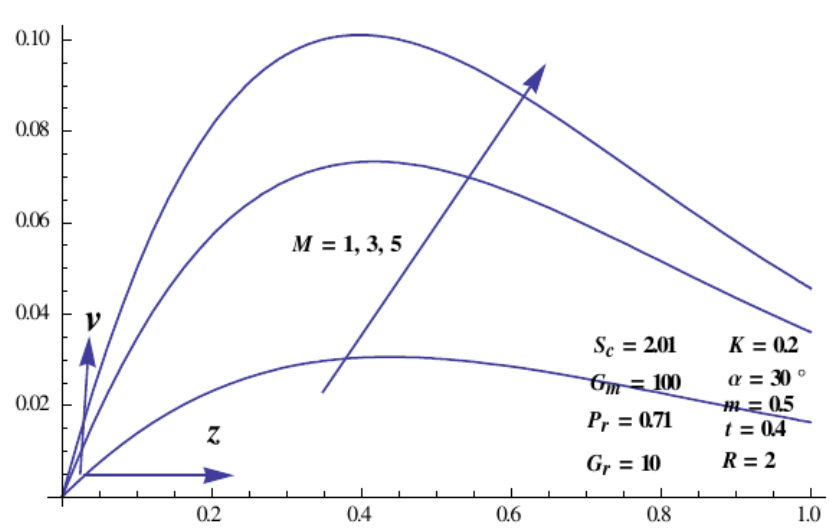

Fig. 16. Velocity $v$ for different values of $M$ 


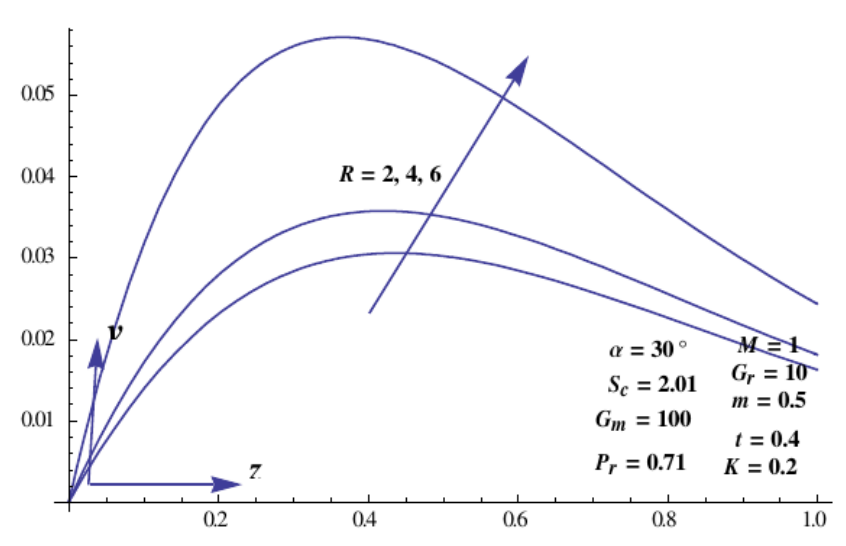

Fig. 17. Velocity $v$ for different values of $R$

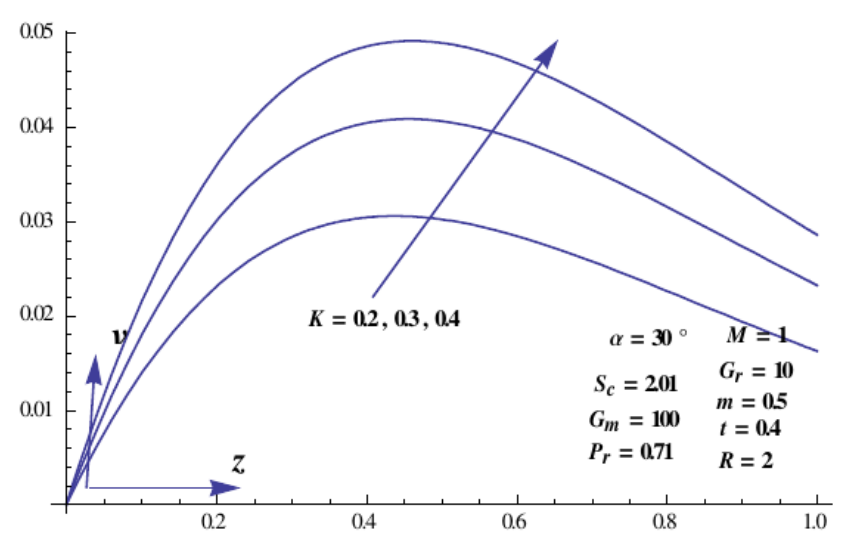

Fig. 18. Velocity $v$ for different values of $K$

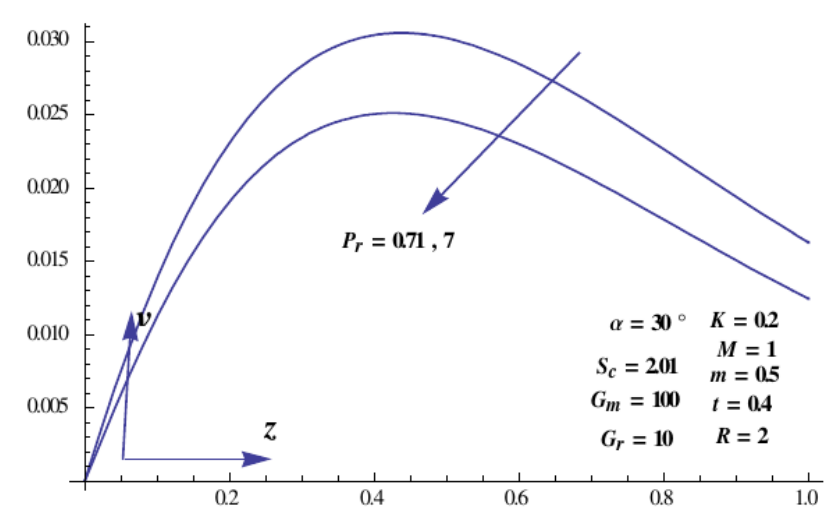

Fig. 19. Velocity $v$ for different values of $P r$

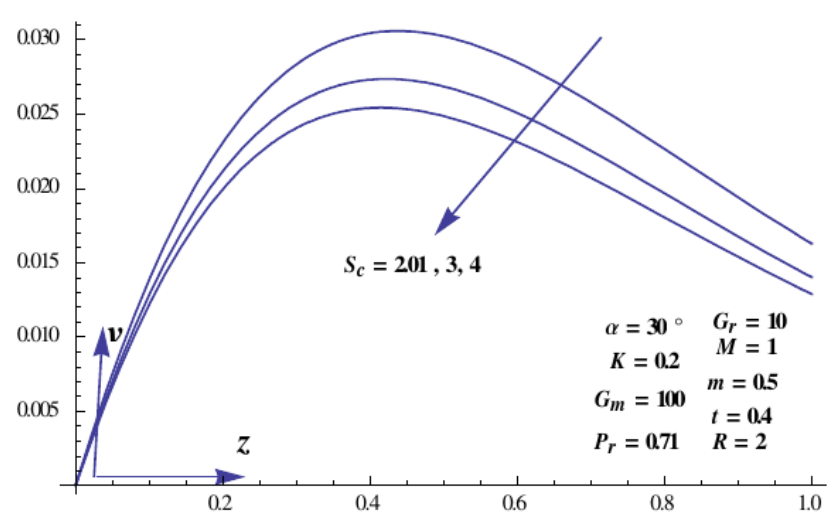

Fig. 20. Velocity $v$ for different values of $S c$

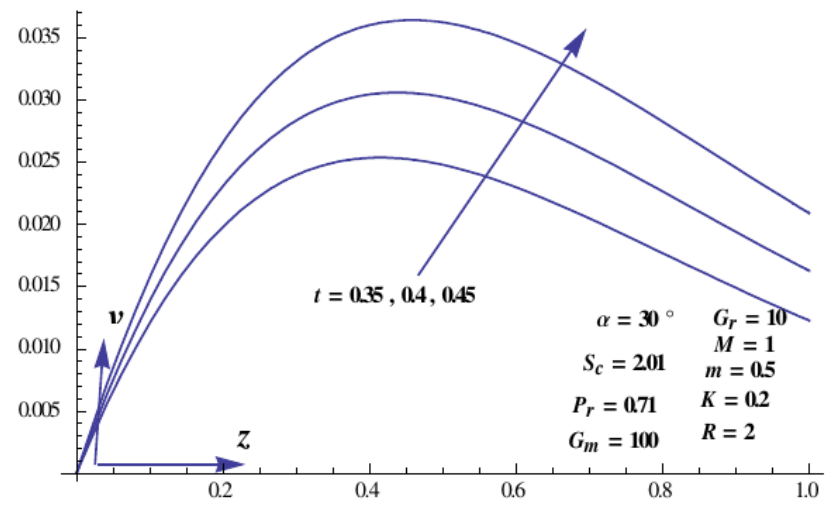

Fig. 21. Velocity $v$ for different values of $t$

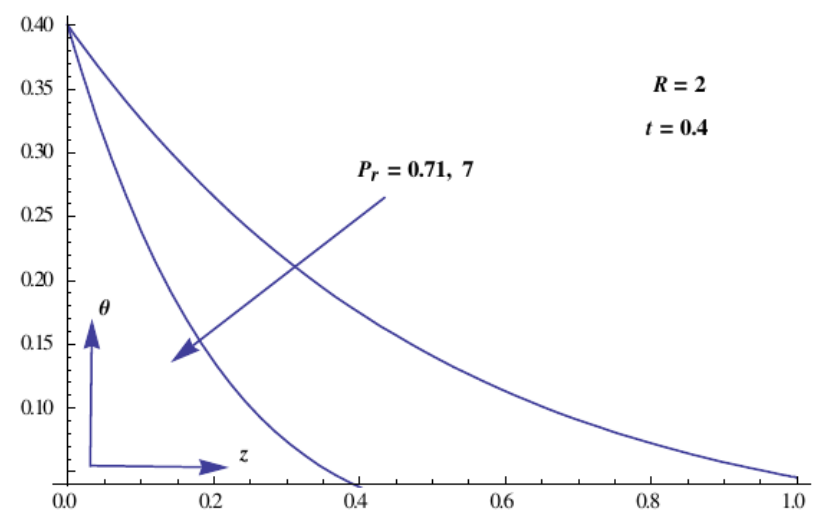

Fig. 22. $\theta$ for different values of $P r$ 


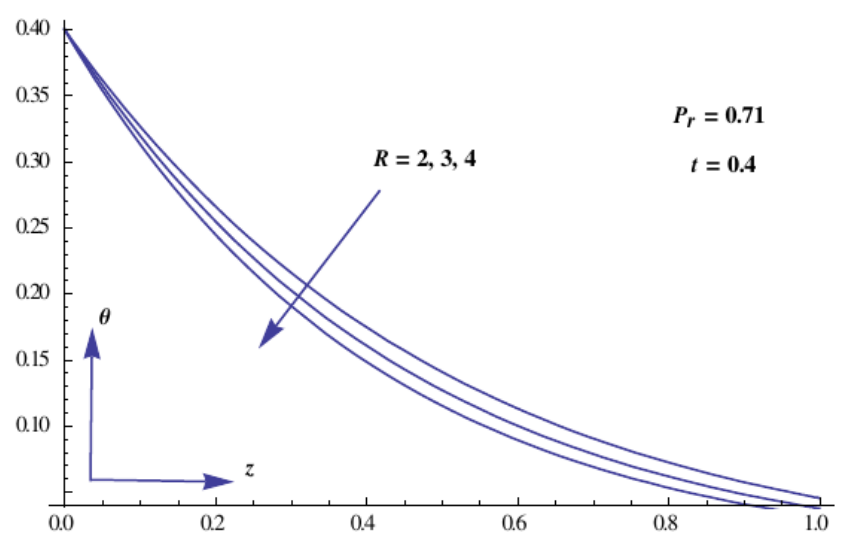

Fig. 23. $\theta$ for different values of $R$

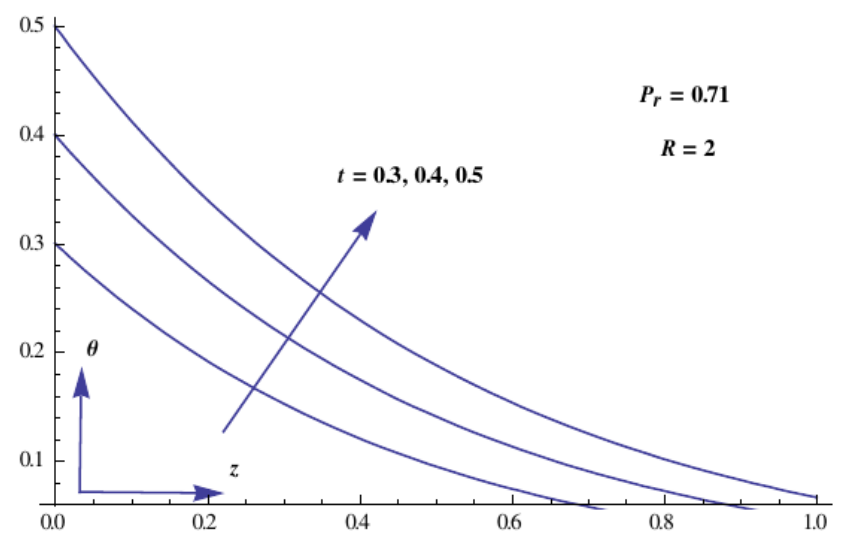

Fig. 24. $\theta$ for different values of $t$

\section{CONCLUSION}

In this paper a theoretical analysis has been done to study the effects of radiation and porosity of the medium on MHD natural convection flow past an impulsively started inclined plate with variable wall temperature and mass diffusion in the presence of Hall current. The results obtained are in agreement with the usual flow. It has been found that the velocity in the boundary layer increases with increasing the values of the radiation parameter and permeability of the medium. It is also observed that the radiation effect and permeability of the medium increase the friction at the plate surface. Also it is found that the radiation effect decreases the values of the Nusselt number. This work can further be extended by considering some more relevant fluid parameters like chemical reaction with rotating system, generation or absorption, etc. The geometry of the model can also be changed to accommodate some MHD flows with important applications.

\section{APPLICATIONS OF THE STUDY}

This study is expected to be useful in understanding the influence of magnetic field and Hall current on astrophysical problems. Moreover, the findings of the research will be useful in improving the devices like Hall accelerators and Hall sensors. It is also useful to study the flow of plasma in MHD power generators, enhanced oil recovery and filtration systems.

\section{Appendix A - symbols}

- $A=\frac{\mathrm{u}_{0}^{2} t}{v}$

- $A_{1}=1+e^{2 \sqrt{a} z}\left(1-A_{18}\right)-A_{17}$,

- $A_{3}=1-e^{2 \sqrt{a} z}\left(1-A_{18}\right)-A_{17}$,

- $A_{4}=-1+A_{19}+A_{30}\left(A_{20}-1\right)$,

- $A_{5}=-1+A_{21}+A_{28}\left(A_{22}-1\right)$,

- $A_{2}=-A_{1}$,

- $A_{6}=-1+A_{23}+A_{26}\left(A_{31}-1\right)$,

- $A_{7}=-1+A_{29}+A_{27}\left(A_{30}-1\right)$,

- $A_{8}=-1+A_{23}+A_{26}\left(A_{31}-1\right)$,

- $A_{9}=-1-A_{24}-A_{28}\left(1-A_{25}\right)$,

- $A_{10}=-A_{9}$,

- $A_{11}=\operatorname{Abs}[z] \cdot \operatorname{Abs}\left[P_{r}\right]$,

- $A_{12}=e^{\frac{a t}{P_{r}-1}-\frac{R t}{P_{r}-1}-z \sqrt{\frac{a P_{r}-R}{P_{r}-1}}}$,

- $A_{13}=e^{\frac{a t}{S_{c}-1}-z \sqrt{\frac{a S_{c}}{S_{c}-1}}}$

- $A_{12}=e^{\frac{a t}{P_{r}-1}-\frac{R t}{P_{r}-1}-\mathrm{Abs}[z] \sqrt{\frac{P_{r}\left(a P_{r}-R\right)}{P_{r}-1}}}$,

- $A_{15}=-1+\operatorname{erf}\left[\frac{z \sqrt{S_{c}}}{2 \sqrt{t}}\right], A_{16}=e^{\mathrm{Abs}[z] \sqrt{P_{r} R}}$,

- $A_{17}=\operatorname{erf}\left[\frac{2 \sqrt{a} t-z}{2 \sqrt{t}}\right]$,

- $A_{18}=\operatorname{erf}\left[\frac{2 \sqrt{a} t+z}{2 \sqrt{t}}\right]$,

- $A_{19}=\operatorname{erf}\left[\frac{z-2 t \sqrt{\frac{a P_{r}-R}{P_{r}-1}}}{2 t}\right]$,

- $A_{20}=\operatorname{erf}\left[\frac{z+2 t \sqrt{\frac{a P_{r}-R}{P_{r}-1}}}{2 t}\right]$,

- $A_{21}=\operatorname{erf}\left[\frac{z-2 t \sqrt{\frac{a S_{c}}{S_{c}-1}}}{2 t}\right]$,

- $A_{22}=\operatorname{erf}\left[\frac{z+2 t \sqrt{\frac{a S_{c}}{S_{c}-1}}}{2 t}\right]$,

- $A_{23}=\operatorname{erf}\left[\frac{\operatorname{Abs}[z] \cdot \operatorname{Abs}\left[P_{r}\right]}{2 \sqrt{t}}-\sqrt{\frac{t R}{P_{r}}}\right]$,

- $A_{24}=\operatorname{erf}\left[\frac{2 t \sqrt{\frac{a}{S_{c}-1}}-2 \sqrt{S_{c}}}{2 t}\right]$,

- $A_{25}=\operatorname{erf}\left[\frac{2 t \sqrt{\frac{a}{S_{c}-1}}+2 \sqrt{S_{c}}}{2 t}\right]$,

- $A_{16}=e^{\mathrm{Abs}[z] \sqrt{P_{r} R}}$

- $A_{27}=e^{2 \mathrm{Abs}[z] \sqrt{\frac{P_{r}\left(a P_{r}-R\right)}{P_{r}-1}}}$,

- $A_{28}=e^{-2 z \sqrt{\frac{a S_{c}}{S_{c}-1}}}$,

- $A_{29}=\operatorname{erf}\left[\frac{\operatorname{Abs}[z] \cdot \operatorname{Abs}\left[P_{r}\right]}{2 \sqrt{t}}-\sqrt{\frac{t\left(R-a P_{r}\right)}{P_{r}\left(1-P_{r}\right)}}\right]$,

- $A_{30}=e^{-2 z \sqrt{\frac{a P_{r}-R}{P_{r}-1}}}$

- $A_{31}=\operatorname{erf}\left[\frac{\operatorname{Abs}[z] \cdot \operatorname{Abs}\left[P_{r}\right]}{2 \sqrt{t}}+\sqrt{\frac{t R}{P_{r}}}\right]$,

- $A_{32}=\operatorname{erf}\left[\frac{\mathrm{Abs}[z] \cdot \operatorname{Abs}\left[P_{r}\right]}{2 \sqrt{t}}+\sqrt{\frac{t\left(R-a P_{r}\right)}{P_{r}\left(1-P_{r}\right)}}\right]$,

- $A_{33}=1+A_{17}+e^{2 \sqrt{a} z} A_{34}, A_{34}=\operatorname{erf} c\left[\frac{2 \sqrt{a} t+z}{2 \sqrt{t}}\right]$. 


\section{Appendix B - nomenclature}

- $a^{*}-$ absorption constant,

- $C$ - species concentration in the fluid,

- $\bar{C}$ - the dimensionless concentration,

- $C_{P}$ - specific heat at constant pressure,

- $C_{w}$ - species concentration at the plate,

- $C_{\infty}$ - the concentration in the fluid far away from the plate,

- $D$ - mass diffusion,

- $g$ - gravity acceleration,

- $G_{m}$ - mass Grashof number,

- $G_{r}$ - thermal Grashof number,

- $k$ - the thermal conductivity,

- $K$ - permeability of the medium,

- $K_{0}$ - the chemical reaction parameter,

- $M$ - the magnetic parameter,

- $m$ - the Hall parameter $\left(m=\omega_{e} \tau_{e}\right)$,

- Pr-Prandtl number,

- $S c-$ Schmidt number,

- $t$-time,

- $T$ - temperature of the fluid,

- $T_{w}$ - Temperature of the plate,

- $T_{\infty}$ - The temperature of the fluid near the plate,

- $u$ - Velocity of the fluid in $x$-direction,

- $\overline{\mathrm{u}}$ - The dimensionless velocity,

- $v$-The velocity of the fluid in $z$-direction,

- $\bar{v}$ - Dimensionless velocity,

- $\omega_{e}$-Cyclotron frequency of electrons,

- $\tau_{e}$ - Electron collision time,

- $\beta=$ volumetric coeff. of thermal expansion,

- $\beta^{*}=$ volumetric coeff. of concentration expansion,

- $v$ - the kinematic viscosity,

- $\rho$ - the fluid density,

- $\sigma$-electrical conductivity,

- $\mu$-the magnetic permeability,

- $\theta$ - the dimensionless temperature,

- $\mu$ - the coefficient of viscosity.

\section{References}

[1] A.N. Armstrong, R. Muthucumaraswamy, MHD flow past a parabolic started vertical plate with variable temperature and mass diffusion, Journal of Mechanical Engineering and Sciences, 7, 1251-1260 (2014).

[2] I.G. Baoku, C.I. Cookey, B.I. Olajuwon, Influence of thermal radiation on a transient MHD Couette flow through a porous medium, Journal of Applied Fluid Mechanics 5(1), 81-87 (2012).

[3] D. Deka, R.K. Deka, Radiation effects on MHD flow past an infinite vertical plate with variable temperature and uniform mass diffusion, JP Journal of Mathematical Sciences, 1(1), 15-29. (2011).

[4] S.K. Guchhait, S. Das, R.N. Jana, Combined effects of Hall current and radiation on MHD free convective flow in a vertical channel with an oscillatory wall temperature, Open Journal of Fluid Dynamics 3, 9-22 (2013).

[5] M.A. Hossain, M.A. Alim, D.A.S. Rees, The effect of radiation in free convection from a porous vertical plate, Int $\mathrm{J}$ Heat and Mass transfer, 42(1), 181-191 (1999).

[6] D. Pal, H. Mondal, Radiation effects on combined convection over a vertical flat plate embedded in a porous medium of variable porosity, Meccanica, 44 133-144 (2009).

[7] A. Raptis, Radiation and free convection flow through a porous medium, Int Comm. Heat Mass Transfer 25(2), 289295 (1998).

[8] A. Raptis, C. Perdikis, Unsteady flow through a highly porous medium in the presence of radiation, Transport Porous Media 57, 171-179 (2004).

[9] U.S. Rajput, P.K. Sahu, Radiation effects on steady hydromagnetic flow of a viscous fluid through a vertical channel in a porous medium with heat generation or absorption, Int $\mathrm{J}$ of Math Archive 2(10), 1-7 (2011).

[10] U.S. Rajput, G. Kumar, Chemical reaction effect on unsteady MHD flow past an impulsively Started oscillating inclined plate with variable temperature and mass diffusion in the presence of Hall current, Applied Research Journal 2(5), 244-253 (2016).

[11] C.S.K. Raju, N. Sandeep, S. Saleem, Effects of induced magnetic field and homogeneous-heterogeneous reactions on stagnation flow of a Casson fluid, Engineering Science and Technology, an International Journal 19, 875-887 (2016).

[12] K. Srihari, Combined effects of Hall current and magnetic field on unsteady flow past a semi-infinite vertical plate with thermal radiation and heat source, Physical Science International Journal 7(2), 73-89 (2015).

[13] M. Thamizhsudar, J. Pandurangan, Combined effects of radiation and Hall current on MHD flow past an exponentially accelerated vertical plate in the presence of rotation, International Journal of Innovative Research in Computer and Communication Engineering 2(12) (2014).

[14] B. Vasu, V.R. Prasad, N.B. Reddy, Radiation and mass transfer effects on transient free convection flow of a dissipative fluid past semi-infinite vertical plate with uniform heat and mass flux, Journal of Applied Fluid Mechanics 4(1), 15-26 (2011).

[15] P. Vyas, N. Srivastava, Radiative MHD flow over a nonisothermal stretching sheet in a porous medium, Applied Mathematical Sciences 4(49-52), 2475-2484 (2010).

[16] H.E. Wilhem, S.H. Choi, Magneto hydrodynamic diffusion flow across homogeneous magnetic field, The Physics of Fluids 21, 1717 (1978). 


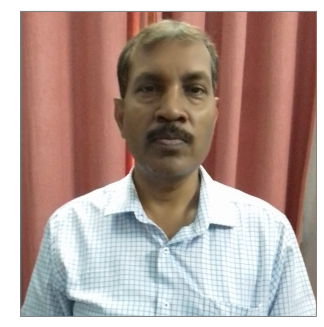

Dr. U.S. Rajput is a faculty member in the department of mathematics and astronomy, University of Lucknow, India. He has more than 25 years of teaching experience at UG and PG levels, and also guided students for $\mathrm{PhD}$ degree. He has published more than 90 research articles. His research areas include MHD flows, Graph Theory and Operations Research.

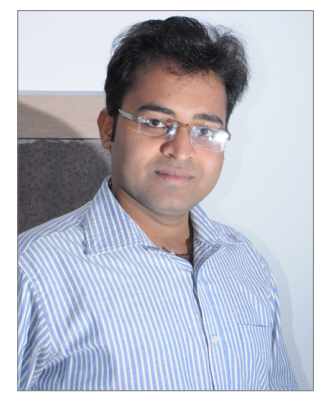

Gaurav Kumar is research student in the department of mathematics and astronomy, University of Lucknow, India. 